\title{
Reconstructing the spatial distribution of the Galactic stellar halo
}

\author{
M. Cignoni ${ }^{1,2} \dagger$, V. Ripepi ${ }^{1}$, M. Marconi ${ }^{1}$, J. M. Alcalá ${ }^{1}$, \\ M. Capaccioli ${ }^{1}$, M. Pannella ${ }^{3}$ and R. Silvotti ${ }^{1}$ \\ ${ }^{1}$ Osservatorio Astronomico Di Capodimonte, Via Moiariello 16, 80131 Napoli, Italy \\ email: cignoni@df.unipi.it \\ ${ }^{2}$ Dipartimento di Astronomia, Università di Bologna, Via Ranzani 1, I-40127 Bologna, Italy \\ ${ }^{3}$ Max-Planck-Institut für extraterrestrische Physik, Giessenbachstrasse, Postfach 1312, \\ D-85741 Garching, Germany
}

\begin{abstract}
The VLT Survey Telescope (VST) is going to offer a unique chance to collect deep and wide field photometry in multi-directions, opening the door to a definitive mapping of the Galactic halo. In this shortcoming scenario, we present a pilot study aimed at recovering the halo stellar density using the Capodimonte Deep Field (OACDF, Alcalá et al. 2004). Turn-off stars are isolated and the relative color-magnitude diagram (CMD) is compared with synthetic CMDs. Our result is consistent with a power law exponent $n \approx 3$ over a range of Galactocentric distances from 8 to $40 \mathrm{kpc}$.
\end{abstract}

Keywords. Galaxy: halo, Galaxy: structure, Galaxy: stellar content, Stars: Hertzsprung-Russell diagram, Methods: statistical

\section{Introduction}

The observed star counts from the OACDF photometric surveył are investigated. This survey covers about 0.5 square degree in the $B, V, R$ optical filters and is located at a high galactic latitude $(l \sim 293, b \sim 50)$, making available a sample of halo stars with low contamination of disk and thick-disk stars.

To derive the spatial distribution of the Galactic halo, one needs to identify stars in a precise evolutionary phase and use them to map the observed CMD density. Turn-off stars have many advantages:

- They can be univocally selected (definitely bluer than halo red giants);

- They are readily distinguishable from the disk stars in the main sequence (only very young and massive disk stars may have similar colors, but they are rare);

- They are numerous, in fact HB stars and red giants are less frequent than turn-off stars of a factor larger than $\approx 100$.

On this basis, we have explored the OACDF color-magnitude diagram selecting turnoff stars by color $(0.3 \lesssim B-V \lesssim 0.6)$. Contamination by galaxies is reduced by choosing objects with stellarity index higher than 0.9 (simultaneously in $B$ and $V$ filters). The adoption of a $V$-magnitude range of 18 to 23 ensures respectively thick disk removal and completeness. Finally, the most probable spatial distribution is obtained by comparing the turn-off density in the observed CMD with that in model CMD.

$\dagger$ Present address: Dipartimento di Astronomia, Via Ranzani 1, I-40127 Bologna, Italy $\ddagger$ Acquired with the Wide Field Imager (WFI) at the ESO 2.2-m telescope. 


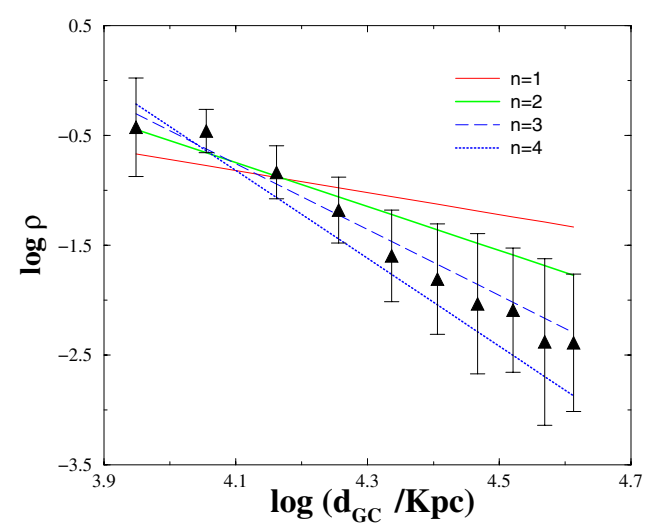

Figure 1. The recovered density distribution. For comparison, different power law densities are also shown (with the labeled exponents).

\section{Method}

Following the Monte Carlo framework, an artificial CMD is populated with a large number of stars randomly built by means of a set of theoretical ingredients (see Cignoni et al. 2006 per a complete description). This synthetic halo population is modeled as originating from a short period of star formation (constant between 10 and $12 \mathrm{Gyr}$ ). Moreover, the metallicity is fixed at $[\mathrm{Fe} / \mathrm{H}] \sim-1.6$ with a Gaussian spread $\left(\sigma_{[\mathrm{Fe} / \mathrm{H}]}=1.0\right)$ and the IMF is a power law with a Salpeter index. For each stellar model of a given mass, age and chemical composition, colors and magnitudes are estimated by interpolating a library of stellar tracks (Pisa Evolutionary Library, Cariulo, Degl'Innocenti \& Castellani 2004). In order to reconstruct the halo structure, this synthetic population is placed at different distances, randomly placing stars in the $j$-th heliocentric interval $\left[d_{\odot, j}, d_{\odot, j}+4 k p c\right] \dagger$. The final product is a base of partial CMDs, each one representing the same population but at different distance moduli. The code searches (through a simplex algorithm) for the linear combination of these basic CMDs which best matches the observed CMD.

\section{Results}

We have combined a star count model with an updated stellar library to reconstruct the Galactic halo stellar density in the direction of the Capodimonte Deep Field. Our results confirm a steep decline. In particular, a power-law index $n \approx 3$ (see Fig. 1) gives a reasonable fit out to $\approx 40 \mathrm{Kpc}$ from the galactic center.

\section{Acknowledgements}

Financial support for this study was provided by MIUR, under the scientific project "On the evolution of stellar systems: fundamental step toward the scientific exploitation of VST" (P.I. Massimo Capaccioli).

\section{References}

Alcalá, J. M. et al. 2004, A\&A, 428, 339

Cariulo, P., Degl'Innocenti, S., \& Castellani 2004, A\&A, 421, 1121

Cignoni, M., Ripepi, V., Marconi, M., Alcalá, J. M., Capaccioli, M., Pannella, M., \& Silvotti, R. 2006, A\&A, DOI: 10.1051/0004-6361:20066598

$\dagger d_{\odot, j}$ is the heliocentric distance of the $j$-th volume bounded by the field of view. 